\title{
EVOLUÇÃO DA MATURAÇÃO DA UVA CABERNET FRANC CONDUZIDA NO SISTEMA LIRA ABERTA
}

\author{
Ripening evolution of Cabernet Franc grapes conducted in the lyre system
}

\author{
Luciano Manfroi ${ }^{1}$, Alberto Miele ${ }^{2}$, Luiz Antenor Rizzon ${ }^{2}$, Carlos I. N. Barradas ${ }^{3}$, Paulo V.D. de Souza ${ }^{3}$
}

\begin{abstract}
RESUMO
Objetivou-se com este trabalho avaliar a evolução da maturação da uva 'Cabernet Franc' conduzida no sistema lira aberta. O trabalho foi realizado durante o ciclo vegetativo de 1995/96, em oito vinhedos instalados em diferentes regiões vitícolas dos municípios de Bento Gonçalves e Monte Belo do Sul, RS. As amostras, formadas por 200 bagas, foram coletadas semanalmente a partir do início da maturação e esmagadas para a extração do mosto. Determinaram-se as variáveis teor de sólidos solúveis totais (TSS), densidade, pH, acidez total, ácido tartárico e ácido málico. Pelos resultados, verificou-se que houve aumentos lineares significativos do teor de sólidos solúveis totais, da densidade, do $\mathrm{pH}$ e das relações TSS/acidez total e ácido tartárico/ácido málico; entretanto, houve diminuição linear e significativa da acidez total, do ácido tartárico e do ácido málico.
\end{abstract}

Termos para indexação: Uva, mosto, sistema de condução, viticultura, maturação.

\begin{abstract}
The objective was to evaluate the ripening evolution of 'Cabernet Franc' grapes conducted in the lyre system. The work was carried out during the vegetative cycle of 1995/96, in eight vineyards located in different viticultural regions of the communes of Bento Gonçalves and Monte Belo do Sul, Brazil. Two hundred berries were collected weekly from the beginning of ripening and were crushed for must extraction. The variables evaluated were ${ }^{\circ} \mathrm{Brix}$, density, $\mathrm{pH}$, total acidity, tartaric and malic acids. Results show that there were significant linear increases of ${ }^{\circ} \mathrm{Brix}$, density, $\mathrm{pH},{ }^{\circ} \mathrm{Brix} /$ total acidity ratio and the sum of tartaric and malic acids; however, there was a decrease of total acidity, tartaric and malic acids.
\end{abstract}

Index terms: Grape, must, training system, viticulture, ripening.

(Recebido para publicação em 11 de setembro de 2003 e aprovado em 15 de outubro de 2003)

\section{INTRODUÇÃO}

A condução da videira tem influência na distribuição e orientação das folhas do dossel vegetativo e na interceptação da radiação solar. A largura do sistema de condução e o nível de desenvolvimento da vegetação condicionam a distribuição dos cachos e das folhas no dossel (CHAMPAGNOL, 1984).

A captação da energia luminosa é feita de forma diferenciada em razão das características inerentes a cada sistema de condução da videira. A penetração da radiação solar no dossel vegetativo favorece a iniciação floral, a fertilidade da gema, o pegamento do fruto e a maturação da uva (CARBONNEAU, 1982). Além disso, influencia o desenvolvimento da planta e a síntese de açúcar (GRIBAUDO et al., 1988), melhorando a produção e a qualidade da uva (SMART, 1985).

A radiação solar na região do cacho é mais importante durante a fase de maturação, pelo seu efeito na composição do mosto, como acidez total, pH e anto- cianinas (TODA et al., 1991). Além disso, folhas expostas ao sol promovem a diferenciação das gemas e favorecem o acúmulo de reservas nas bagas e a salificação dos ácidos orgânicos (RIDOMI e MORETTI, 1996). Por outro lado, dosséis vegetativos densos proporcionam menor produtividade da videira e menor qualidade do fruto (SMART, 1985).

A condução da videira no sistema lira aberta apresenta como benefícios baixo custo de produção, melhor regularidade na produção, maturação da uva e potencial qualitativo dos vinhos (CARBONNEAU, 1991). Na Serra Gaúcha, o sistema de condução predominante é a latada que, se não houver um adequado manejo do dossel vegetativo, geralmente apresenta microclima desfavorável à qualidade da uva. Há poucos anos, alguns viticultores formaram vinhedos conduzidos no sistema lira aberta como alternativa à tradicional latada, mas ainda não há avaliação desse sistema. Em face disso, conduziu-se este trabalho, 
que avaliou a evolução dos fatores que caracterizam o açúcar e a acidez da uva 'Cabernet Franc' conduzida em lira aberta na principal região vitivinícola do País.

\section{MATERIAL E MÉTODOS}

O trabalho foi realizado no ciclo vegetativo de 1995/96, em oito vinhedos de Cabernet Franc localizados nos municípios de Bento Gonçalves (comunidades de São Valentim, Tuiuti e Pinto Bandeira) e Monte Belo do Sul, RS.

Os vinhedos foram formados em 1989, utilizando mudas de 'Cabernet Franc' enxertadas sobre o porta-enxerto SO4. As videiras foram conduzidas em lira aberta, sistema que se caracteriza pela condução das videiras em dois planos verticais inclinados, mantidos por três ou quatro fios em cada plano. O espaçamento variou de $1,00 \mathrm{~m}$ a $1,35 \mathrm{~m}$ entre plantas e de $2,50 \mathrm{~m}$ a 3,10 $\mathrm{m}$ entre fileiras. A poda seca foi a mista, deixando-se, em média, 4,3 varas/planta com 5,3 gemas/vara e 11,9 esporões/planta com 1,3 gema/esporão, ou seja, 38,3 gemas/planta.

A partir da mudança de cor, que teve início dia 9 de janeiro de 1996, foram feitas semanalmente cinco amostragens de uva nos dias 10, 17, 24 e 31 de janeiro e 7 de fevereiro. Cada amostra constou de 200 bagas por vinhedo, sendo coletada uma baga por cacho em todos os quadrantes, aleatoriamente, até o total de 20 bagas por planta. As amostras foram levadas ao laboratório e esmagadas para a extração do mosto que foi, a seguir, centrifugado.

O teor de sólidos solúveis totais, expresso em ${ }^{\circ}$ Brix, foi determinado por um refratômetro digital de bancada com controle automático de temperatura; a densidade, por um densímetro digital, marca Anton Paar, modelo DMA-45; a acidez total, expressa em ácido tartárico, pela titulação do mosto com Na$\mathrm{OH} 0,1 \mathrm{~N}$, utilizando o azul de bromotimol como indicador; o $\mathrm{pH}$, com um potenciômetro digital, marca
CORNING, modelo 125, equipado com eletrodo de vidro e calibrado com solução-padrão de pH 3,0 e pH 4,0 .

Os ácidos tartárico e málico foram analisados por cromatografia líquida de alta performance, utilizando um aparelho equipado com um injetor Rheodyne de $20 \mu \mathrm{L}$ de volume, um detector UV/VIS e uma coluna VARIAN MCH-NCAP-5 (AUGUSTE, 1979).

Os dados obtidos foram avaliados pela análise de regressão polinomial, utilizando o programa estatístico Sanest.

\section{RESULTADOS E DISCUSSÃO}

A evolução da maturação da uva Cabernet Franc conduzida em lira aberta mostrou que houve aumento linear significativo no teor de sólidos solúveis (Figura 1). O valor do teor de sólidos solúveis, estimado pela equação de regressão polinomial, variou de 11,96, no início da maturação, a 17,50, na maturação, ou seja, houve um aumento médio de 0,198 sólidos solúveis/dia. No início da maturação, o teor de sólidos solúveis foi influenciado pelo alto teor em ácidos orgânicos presentes na baga. Mas, com a evolução da maturação, a participação desses ácidos tornou-se menor, e a dos açúcares, maior, em decorrência da degradação dos ácidos tartárico e málico e do aumento da síntese de sacarose pela folha.

$\mathrm{Da}$ mesma forma que o teor de sólidos solúveis, a densidade teve aumento linear significativo (Figura 2), pois ela tem relação direta com a concentração de açúcares no mosto. Os valores variaram de 1,0514 no início da maturação a 1,0749 na maturação da uva, com aumento médio de 0,00084/dia. À medida que ocorre a maturação, a densidade torna-se uma medida mais real da concentração de açúcares do mosto. 


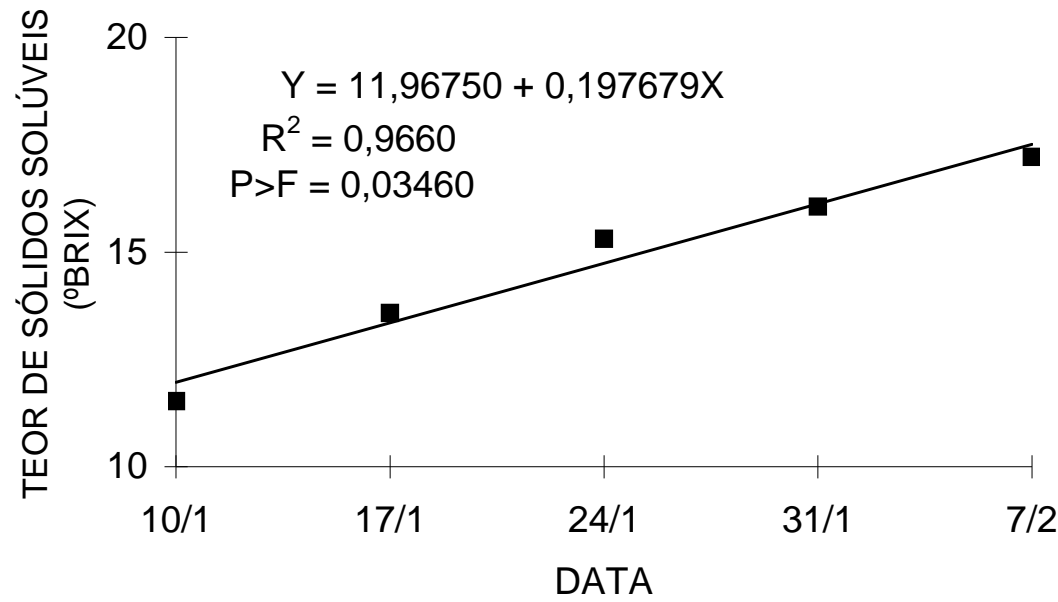

FIGURA 1 - Evolução do teor de sólidos solúveis do mosto da cv. Cabernet Franc conduzida no sistema lira aberta. Ciclo vegetativo 1995/96.

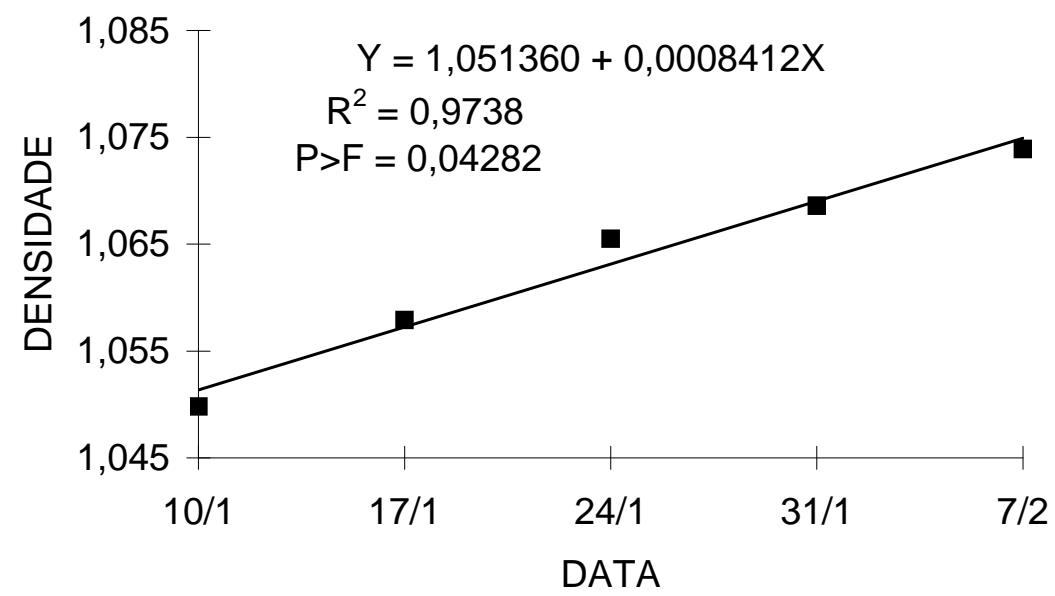

FIGURA 2 - Evolução da densidade do mosto da cv. Cabernet Franc conduzida no sistema lira aberta. Ciclo vegetativo $1995 / 96$.

$\mathrm{O}$ pH teve um aumento linear, passando de 2,74 no início da maturação a 3,18 na maturação (Figura 3); houve, então, um aumento médio de 0,016/dia. O aumento no $\mathrm{pH}$ foi devido à diminuição nas concentrações dos ácidos tartárico e málico, à salificação dos ácidos orgânicos, especialmente do tartárico, e ao aumento do cátion potássio.

A acidez total decresceu linearmente e significativamente durante a maturação da uva (Figura 4), pois no início da maturação, a acidez total estimada do mos- to foi de 281 meq $\mathrm{L}^{-1}$ e na maturação, de $85 \mathrm{meq} \mathrm{L}^{-1}$. Houve, portanto, uma diminuição média de $7,0 \mathrm{meq} \mathrm{L}^{-}$ $1 /$ dia. A acidez na baga aumenta da frutificação efetiva do fruto até o início da maturação, diminuindo dessa fase até a maturação da uva (RIBÉREAU-GAYON et al., 1986). A diminuição da concentração dos ácidos orgânicos durante a evolução da maturação é devida à diluição do mosto pela entrada de água no fruto, transportada no xilema pela mobilização de bases que neutralizam os ácidos orgânicos e pelo processo respiratório. 


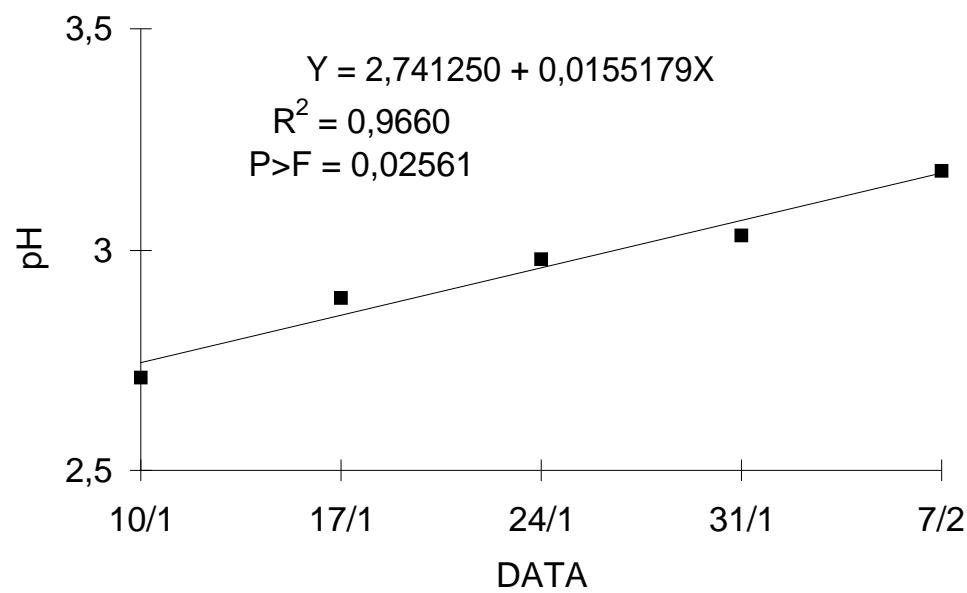

FIGURA 3 - Evolução do pH do mosto da cv. Cabernet Franc conduzida no sistema lira aberta. Ciclo vegetativo 1995/96.

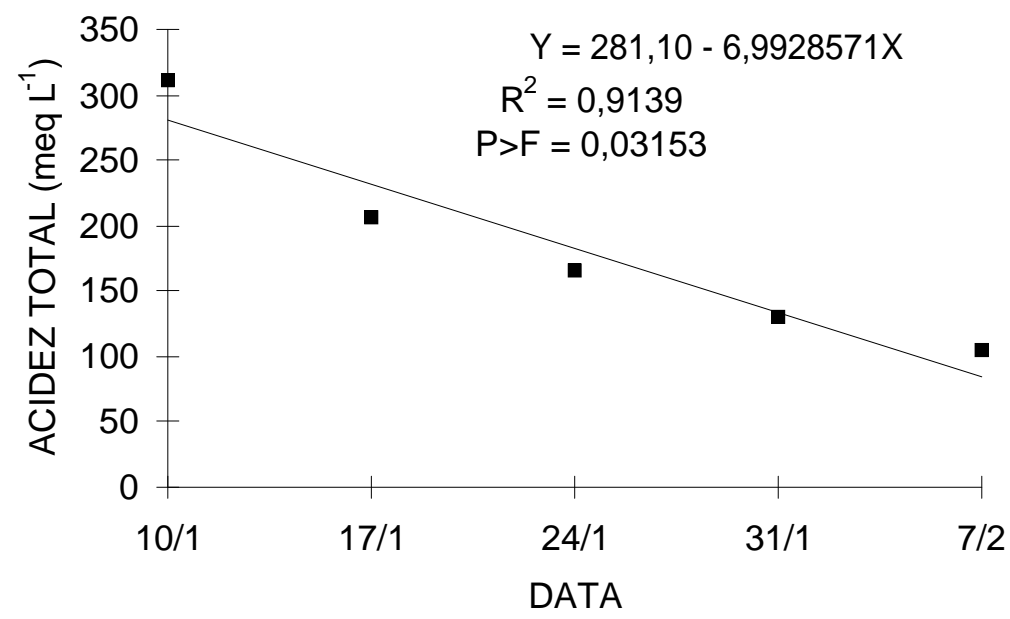

FIGURA 4 - Evolução da acidez total do mosto da cv. Cabernet Franc conduzida no sistema lira aberta. Ciclo vegetativo 1995/96.

Em função da evolução inversa dos fatores que influem nas variáveis relacionadas ao açúcar e à acidez, a relação TSS/acidez total aumentou de forma linear e significativa durante a maturação. Essa relação foi de 4,87 no início da maturação e de 22,12 na maturação (Figura 5), com aumento médio de 0,616/dia. A relação TSS/acidez total é uma das variáveis que podem caracterizar as cultivares numa determinada região. No entanto, a utilização dessa relação como índice de maturação da uva deve ser feita com cautela, pois aumentos na concentração de açúcar nem sempre correspondem à igual redução da acidez total.
A concentração de ácido tartárico diminuiu linearmente do início da maturação à maturação da uva, passando de 124,8 meq L ${ }^{-1}$ a 84,6 meq L $^{-1}$ (Figura 6), o que indica uma diminuição média de 1,44 meq $\mathrm{L}^{-1} /$ dia. Em estudos anteriores realizados na Serra Gaúcha, constatou-se que a evolução do ácido tartárico passou de 99 meq $\mathrm{L}^{-1}$ a 67 meq $\mathrm{L}^{-1}$ e seu teor foi maior na cv. Cabernet Franc que nas demais cultivares viníferas avaliadas (GUERRA, 1989). Os ácidos orgânicos são sintetizados pela respiração celular que ocorre nas partes verdes das plantas; portanto, estão presentes no estado livre ou salificado em todos os órgãos da videira. A 
concentração de ácidos orgânicos livres no fruto geralmente aumenta da periferia para a parte interna, podendo variar conforme a espécie, a interação mesoclima/solo, as práticas culturais e a maturação da uva. O teor de ácido tartárico tem grande relação com a temperatura e, sobretudo, com a circulação de água na planta. Períodos quentes e secos contribuem para a redução do teor de ácido tartárico da uva. Por outro lado, precipitações contínuas por alguns dias favorecem o aumento do ácido tartárico (RIBÉREAUGAYON et al., 1986).

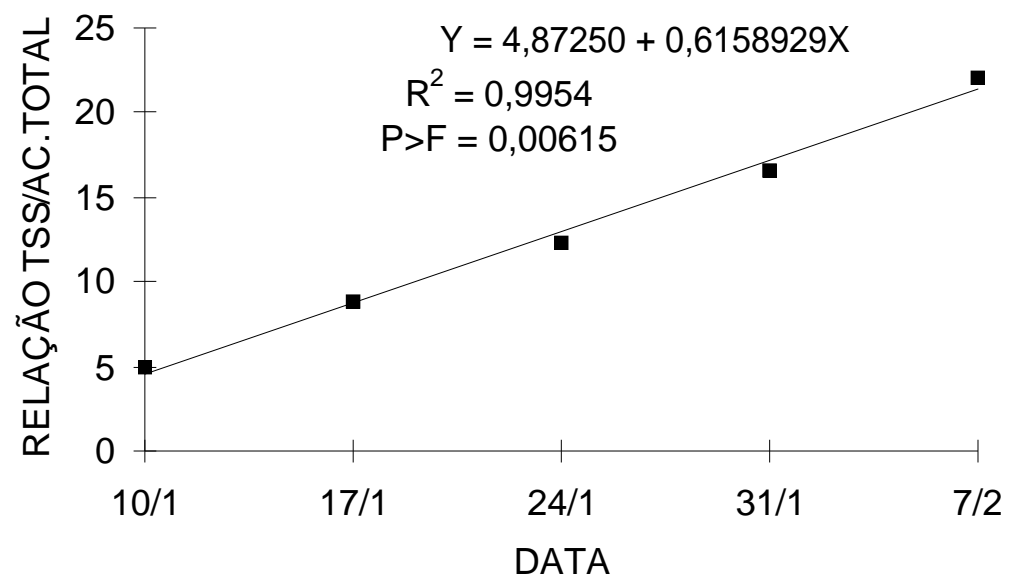

FIGURA 5 - Evolução da relação TSS/acidez total do mosto da cv. Cabernet Franc conduzida no sistema lira aberta. Ciclo vegetativo 1995/96.

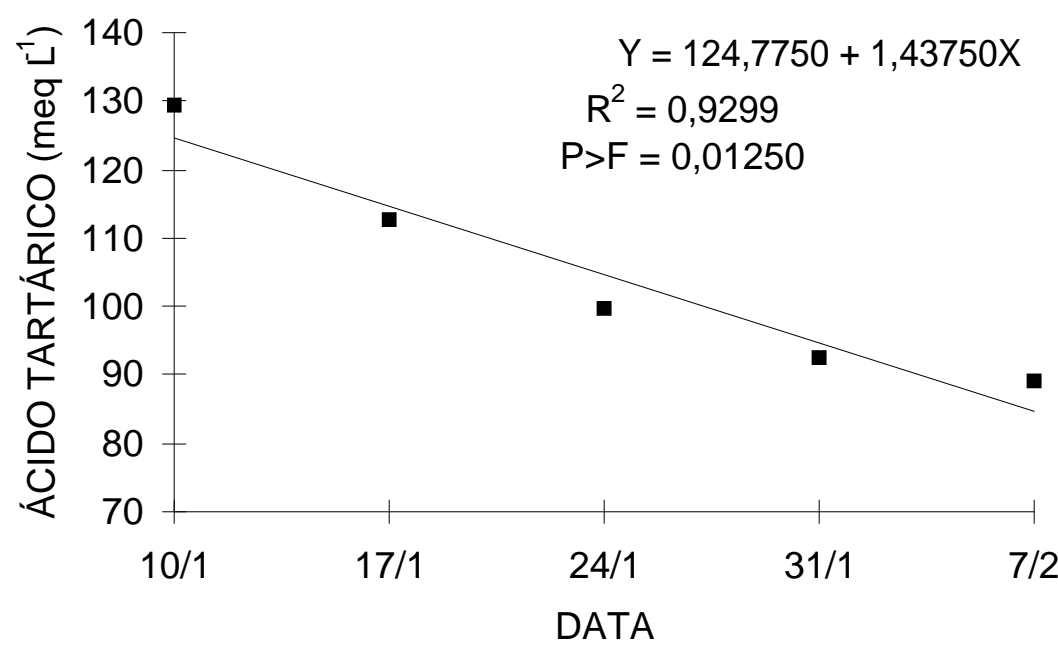

FIGURA 6 - Evolução do ácido tartárico do mosto da cv. Cabernet Franc conduzida no sistema lira aberta. Ciclo vegetativo 1995/96. 
A curva de evolução do ácido málico foi similar à do ácido tartárico, porém, mais acentuada, pois no início da maturação sua concentração foi de 186,9 meq $\mathrm{L}^{-1}$ e na maturação, 46,3 meq L $\mathrm{L}^{-1}$ (Figura 7). Portanto, houve uma diminuição média de $5,02 \mathrm{meq} \mathrm{L}^{-1} /$ dia. O ácido málico é encontrado nas folhas e nos frutos. É um ácido fraco e facilmente metabolizado (PEYNAUD, 1985), diminuindo acentuadamente na cv. Cabernet Sauvignon após a mudança de cor (CRIPPEN e MORRISON, 1986). A evolução do ácido málico de- pende principalmente da combustão respiratória e de sua migração no floema da videira.

No início da maturação, a soma dos ácidos tartárico e málico foi de $311,7 \mathrm{meq} \mathrm{L}^{-1}$, reduzindo linearmente sua concentração na maturação para $130,8 \mathrm{meq} \mathrm{L}^{-1}$ (Figura 8). A diminuição média foi de $6,46 \mathrm{meq}^{-1} / \mathrm{dia}$. A relação ácido tartárico/ácido málico foi de 0,56 no início da maturação e de 1,58 na maturação da uva Cabernet Franc, sendo essa diferença causada pela maior degradação do ácido málico (Figura 9).

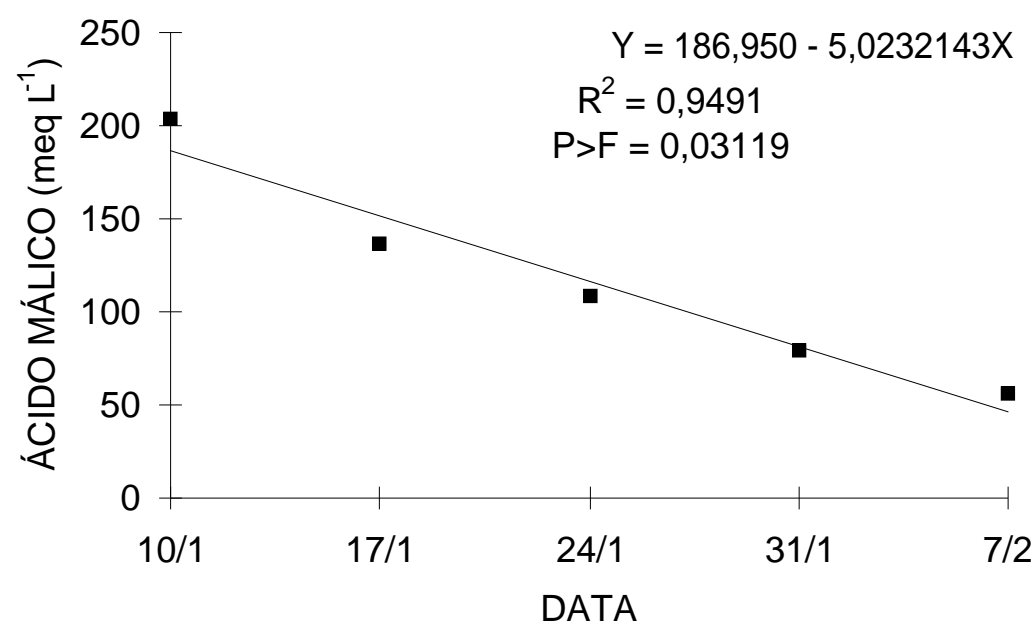

FIGURA 7 - Evolução do ácido málico do mosto da cv. Cabernet Franc conduzida no sistema lira aberta. Ciclo vegetativo 1995/96.

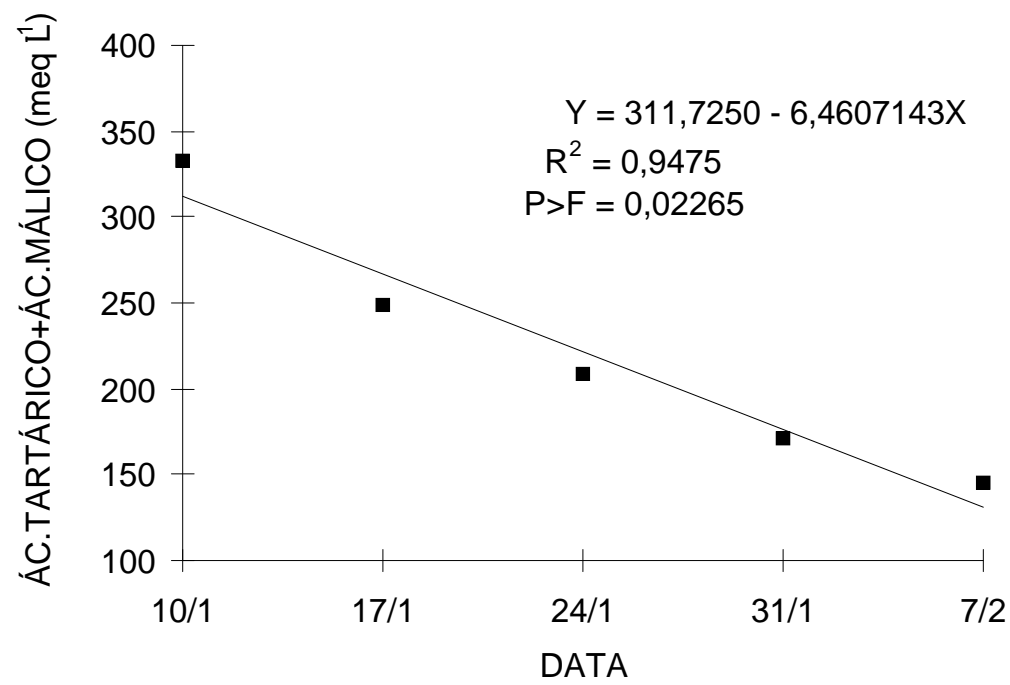

FIGURA 8 - Evolução do ácido tartárico + ácido málico do mosto da cv. Cabernet Franc conduzida no sistema lira aberta. Ciclo vegetativo 1995/96. 


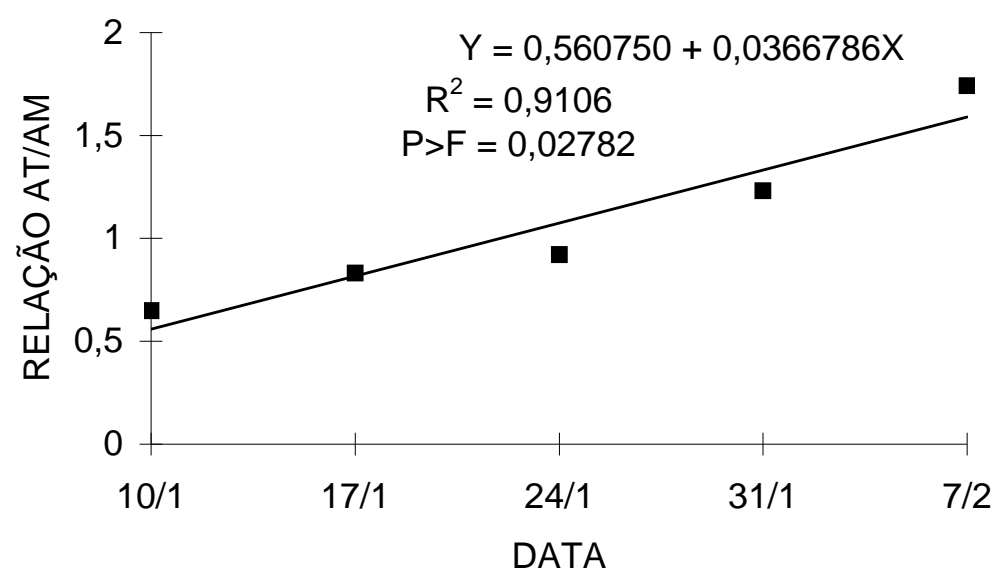

FIGURA 9 - Evolução da relação ácido tartárico/ácido málico do mosto da cv. Cabernet Franc conduzida no sistema lira aberta. Ciclo vegetativo 1995/96.

\section{CONCLUSÕES}

A evolução da maturação da uva 'Cabernet Franc' conduzida em lira aberta na Serra Gaúcha caracterizou-se por aumentos lineares do teor de sólidos solúveis, densidade, $\mathrm{pH}$, relação TSS/acidez total e relação ácido tartárico/ácido málico e por diminuições também lineares da acidez total, ácido tartárico, ácido málico e a soma do ácido tartárico e ácido málico, evidenciando que esse sistema de condução é viável para produção da uva 'Cabernet Franc'.

\section{REFERÊNCIAS BIBLIOGRÁFICAS}

AUGUSTE, M. H. Application de la chromatographie en phase liquide à haute pression à l'analyse des moûts et des vins. 1979. $135 \mathrm{f}$. Tese (Doutorado em Viticultura-Enologia) - Institut d'Oenologie, Université de Bordeaux II, Talence, 1979.

CARBONNEAU, A. Apports biologiques récents à l'étude des systèmes de conduite. Bulletin de l'O.I.V., Paris, v. 55, n. 614, p. 273-285, 1982.

CARBONNEAU, A. Interactions "terroirs x PG x cépages $x$ techniques de culture". Rivista di Viticoltura e di Enologia, Treviso, v. 44, n. 4, p. 53-57, 1991.

CHAMPAGNOL, F. Éléments de physiologie de la vigne et de viticulture générale. Montpellier: Déhan, 1984. $351 \mathrm{p}$.
CRIPPEN JÚNIOR, D. D.; MORRISON, J. C. The effects of sun exposure on the compositional development of Cabernet Sauvignon berries. American Journal of Enology and Viticulture, Davis, v. 37, n. 4, p. 235-242, 1986.

GRIBAUDO, I.; SCHUBERT, A.; NOVELLO, V. Produzione di sostanza secca ed intercettazione di energia luminosa nel vitigno "Cortese" in quattro forme di allevamento. Vignevini, Bologna, v. 15, n. 3, p. 5356,1988 .

GUERRA, C. C. Evolução dos ácidos tartárico e málico durante a maturação de uvas do Rio Grande do Sul. 1989. 98 f. Dissertação (Mestrado em Ciência e Tecnologia de Alimentos) - Universidade Federal de Santa Maria, Santa Maria, 1989.

PEYNAUD, É. Enología e tecnica del vino. 2. ed. Brescia: AEB, 1985. 254 p.

RIBÉREAU-GAYON, J.; PEYNAUD, É.; RIBÉREAU-GAYON, P.; SUDRAUD, P. Carattere dei vini, Maturazione dell'uva, Lieviti e batteri: trattato di scienza e tecnica enologica. Brescia: AEB, 1986. v. 2,424 p.

RIDOMI, A.; MORETTI, G. Comportamento della cv. Cabernet Franc (Vitis vinifera L.) allevata in cinque combinazioni d'innesto e con due sesti d'impianto. Vignevini, Bologna, v. 23, n. 7-8, p. 3-8, 1996. 
SMART, R. E. Principles of grapevine canopy microclimate manipulation with implications for yield and quality. A review. American Journal of Enology and Viticulture, Davis, v. 36, n. 3, p. 230239, 1985.
TODA, F. M. de; SANCHA, J. C.; LLOP, E. Estudio comparado del microclima luminoso en los sistemas de conducción en vaso y espaldera en Rioja. Rivista di Viticoltura e di Enologia, Treviso, v. 44, n. 4, p. 149156, 1991. 